ISSN: 1130-3743 - e-ISSN: 2386-5660

DOI: http://dx.doi.org/10.14201/teoredu2922539

\title{
EL DIÁLOGO COMO APUESTA ÉTICA EN LAS ESCUELAS PLURALISTAS
}

\section{Dialogue as an ethical commitment in pluralistic schools}

\section{Le dialogue comme un engagement éthique dans les écoles pluralistes}

\author{
Juan Eduardo ORTIZ LÓPEZ \\ Universidad Bernardo O'Higgins. Centro de Estudios Políticos, \\ Culturales y Sociales de América Latina. C/ Fábrica, 1990. Comuna de Santiago. \\ Santiago de Chile.juortizl@uc.cl
}

Fecha de recepción: enero de 2017

Fecha de aceptación: marzo de 2017

\section{RESUMEN}

El siguiente artículo tiene por objetivo conceptualizar el diálogo como estrategia enseñable que permite generar procesos de escucha activa, de resolución de problemas, de formación ética y aceptación del otro dentro del contexto de las escuelas pluralistas. Se identifica el diálogo como un espacio clave para la reflexión y para la comunicación, comprendiendo que éste conlleva particularidades como la resistencia o la asimetría en términos de poder. Por otro lado, las escuelas pluralistas basan su estructura en la convivencia de diversas culturas, que se ven beneficiadas por el diálogo entre ellas. No obstante, es necesario comprender que la validación y aceptación de la cultura del otro también transita por la perspectiva ética desde la cual se origina la comunicación entre los actores. En suma, el diálogo constituye una estrategia crucial para la diversidad y la convivencia en las escuelas democráticas y pluralistas.

Palabras clave: diálogo; ética; pluralismo; convivencia. 


\section{SUMMARY}

The following article aims to conceptualise dialogue as a teachable strategy that allows to generate active processes of listening, problem-solving tasks, ethical formation, and acceptance of the other within the context of pluralist schools. Dialogue is identified as a key space for reflection and communication, comprehending that this entails particularities such as resistance and asymmetry in terms of power. On the other hand, pluralist schools base their structure on the coexistence of diverse cultures which are mutually benefited through dialogue. Nevertheless, it is necessary to understand that the others' culture's validation and acceptance move through the ethical perspective from which the communication between the actors occur. All in all, dialogue constitutes a crucial strategy for diversity and coexistence in the pluralist and democratic schools.

Key words: dialogue; ethics; pluralism; coexistence.

\section{SOMMAIRE}

L'article suivant vise à conceptualiser le dialogue comme stratégie enseignable qui génère des processus de l'écoute active, résolution de problèmes, la formation à l'éthique et l'acceptation des autres dans le contexte des écoles pluralistes. Il identifie le dialogue comme un espace clé pour la réflexion et pour la communication, la compréhension de ce que cela implique notamment de la résistance ou de l'asymétrie en termes de puissance. D'autre part, la structure de l'école pluraliste fondée sur la coexistence de différentes cultures qui bénéficient du dialogue entre eux. Cependant, il est nécessaire de comprendre que la validation et l'acceptation de la culture de l'autre passe aussi par la perspective éthique à partir de laquelle la communication entre les acteurs est originaire. En bref, le dialogue est une stratégie cruciale pour la diversité et la coexistence dans les écoles démocratiques et pluralistes.

Mots clés: dialogue; éthique; pluralisme; coexistence.

\section{INTRODUCCIÓN}

Dentro del campo de las complejas relaciones humanas, el diálogo se nos presenta como campo común de la convivencia y del ejercicio de la tolerancia en pos de las libertades individuales de los demás. En el presente trabajo se pretende dar cuenta del diálogo como basamento en la generación de significados comunes y sociales, así como la vivencia cotidiana de la democracia y la ciudadanía. En una primera parte, se da cuenta del concepto y sus implicancias. En la segunda parte, se consideran aspectos éticos que se ponen en juego en la creación del espacio común. En la tercera parte, se han tomado en cuenta a las relaciones dialógicas como pilar de la convivencia escolar, para una formación en los principios democráticos de la sociedad actual. En la cuarta parte, se tratan cuestiones relativas al 
currículo nacional chileno, específicamente poniendo atención a los objetivos fundamentales transversales. Y en una quinta parte, se intentará dar cuenta de cómo reconstruir el diálogo en contextos de resistencia.

\section{CONCEPTO}

Con el devenir de los avances tecnológicos e informáticos, nuestras sociedades han experimentado un cambio profundo. Las conexiones que se establecen entre los distintos seres humanos son cada día más rápidas y cercanas, más inmediatas. La información está al alcance de un solo clic. Basta con introducir una palabra en buscadores en Internet y obtendremos un sinnúmero de resultados. Además, podemos comunicarnos a través de teléfonos móviles o mensajería en la Web. Sin embargo, los seres humanos seguimos necesitando el contacto real con el otro, hecho que ha permitido la misma supervivencia de la especie. Y es aquí, en este mundo cada vez más pequeño, interconectado y multicultural, en donde se suscitan conflictos y prejuicios.

Dentro de este contexto de nuestras sociedades informatizadas e interconectadas, el diálogo se nos presenta como eje organizador de la convivencia humana y con su entorno. Nuestras sociedades democráticas y multiculturales deberían basarse en un diálogo permanente. En consecuencia, nuestras escuelas también deberían hacerse cargo de éste a través de todas sus instancias formadoras y, por qué no, transformadoras. Es decir, las acciones dialogantes y dialógicas que establezca la escuela podrían permitir el desarrollo de nuevas transformaciones en los seres humanos, así como el desarrollo de esta misma capacidad. En este sentido, es innegable que la pedagogía tiene un llamado de carácter hermenéutico, es decir, ha de esforzarse por comprender al otro orientando dicha comprensión a entender la mente del otro y al otro en sí mismo, por lo que los procesos educativos deben responder a este llamado tanto en lo procedimental como en sus principios (Plante, 2013). De este modo, el desarrollo de las libertades individuales y el bien común no se enfrentarían en posiciones antagónicas, sino que más bien se negociarían, así como se negocian los significados en la comunicación.

Conviene, entonces, explicar lo que se entenderá por diálogo en este artículo. La raíz etimológica de la palabra proviene del griego "dia» y «logos», en donde la primera significa "a través de» y la segunda «verbo, palabra, conocimiento». Desde su etimología la palabra ya nos oferta una riqueza de interpretaciones. Implica a los sujetos y sus representaciones, sus conceptos, su lenguaje y, por tanto, su cultura. Establece una conexión entre los sujetos por medio de la palabra, lo que sugiere, además, la posibilidad de relacionarnos e involucrarnos con el otro.

La conceptualización del diálogo que establece Bohm (1997) es reveladora, en donde lo define como «una corriente de significado que fluye entre, dentro y a través de los implicados, y este significado compartido es el aglutinante, el cemento que sostiene los vínculos entre las personas y las sociedades» (Bohm, 1997, 29). Es decir, no sólo se trata de un canal por donde se transmiten mensajes, es un 
sistema de interacciones comunicativas que se relacionan entre sí, generando así significados propios de las relaciones humanas y de las visiones particulares en pos de la creación de espacios comunes. Además, se le establece como el sostén de los vínculos humanos, o sea, a través del diálogo seríamos capaces de generar afectos, convirtiéndose éste en una experiencia sensorial y emocional. En este sentido, el diálogo se manifiesta como una experiencia transformadora y generadora en la vida de los seres humanos.

Es necesario no confundir el diálogo con un traspaso de la propia información al otro. Si ésa fuese nuestra intención, podríamos caer en la persuasión, la que no tiene por qué tener un carácter peyorativo, pero que no guarda relación directa con el objetivo del diálogo. La persuasión tiene un carácter individual, en donde el "yo" manifiesta sus puntos de vista, "sus verdades» y trata de convencer al otro de ello. El diálogo, sin embargo, tiene un carácter colectivo, es decir, "no está vinculado a la verdad sino al significado y éste no es resultado de una opción individual sino de una construcción social» (Bohm, 1997, 33). El diálogo, por tanto, es forma básica y fundamental de la cultura, utilizando para ello el lenguaje, el que, a su vez, también es cultura (Carey, 1989; Martin y Nakayama, 2010). El diálogo, entonces, se convierte tanto en significante como en significado, como un espacio común en donde significar y desde donde se comparten los significados. En consecuencia, el diálogo se convierte en herramienta de cambio y de formación de consensos básicos (Skidmore y Murakami, 2016). Es por ello que el diálogo debería ser eje organizador de la convivencia ciudadana en las escuelas de sociedades democráticas y plurales. Por tanto, es fundamental realizar una elección ética respecto de qué es "lo común" y qué es "lo individual", pero teniendo en mente que una ética basada en el diálogo supone igualdad entre los interlocutores, con iguales derechos, quienes, partiendo de sus intereses subjetivos y mediante un procedimiento adecuado de tolerancia, pueden encontrar normas morales universales de consenso racional, para resolver conflictos en una sociedad. En este sentido, la educación dialógica imbrica la formación ético-valórica, lo que supone, primeramente, establecer la perspectiva ética y axiológica desde la cual se establece el diálogo.

Por otro lado, el diálogo constituye una posibilidad de enfrentar las dificultades de lo que hoy conocemos como «sociedades líquidas», especialmente si consideramos que el diálogo se relaciona con los distintos tipos de capital (Bourdieu, 2011) que un niño o joven ostenta (Guichard, 2013), por lo que es necesaria la interacción de manera tal que las personas puedan transitar de un estado de capital a otro. En este sentido, es posible advertir que el diálogo tiene un carácter reproductor, pero también transformador.

\section{MíNIMOS y MÁXIMOS ÉTICOS, El POliteÍSMO AXIOLÓGICO Y EL PLURALISMO}

El desarrollo de la ética en la escuela de la sociedad actual requiere de comprender las nociones de mínimos y máximos éticos o morales (Rodríguez y Peralta, 2013; Cortina, 2000, 2003, 2010; Vásquez, 2011). El concepto de máximo 
moral guarda relación con las construcciones valóricas que realiza un determinado individuo respecto de su propio lugar en el mundo y la visión que éste posee del mismo. Tiene que ver con una forma de concebir los valores desde un punto de vista individual, subjetivo, en el sentido de que las propias creencias y juicios son considerados como válidos, pero los que no necesariamente representan los valores de los otros (Cortina, 2010). Es en este sentido en el que nos encontramos con la noción de mínimos morales, los que serían construcciones valóricas comunes para los miembros de una determinada sociedad (Vásquez, 2011). Los mínimos morales establecen un margen axiológico común para todos, siendo el primer valor compartido el respeto de las diferencias (Cortina, 2000). Debido a que convivimos en una sociedad plural y democrática, nuestros máximos morales podrían verse confrontados con los de los otros y producir una suerte de politeísmo axiológico en donde no se intente buscar puntos comunes que faciliten la convivencia (Román, 2000). El politeísmo axiológico tiene que ver con una concepción del ejercicio de la libertad individual en términos de lo que el sujeto determina o cree como máximo. Tiene que ver con el desarrollo de las máximas kantianas en el que la propia decisión respecto de lo que debe ser «bueno» es válida y que cualquier intento por regular esa convicción es un atropello a las libertades (Cortina, 2010). Claramente, es posible notar que este politeísmo impide la convivencia entre los diferentes seres humanos de una sociedad, puesto que las máximas de unos se contraponen a las de los otros, y si este tipo de ejercicio de la propia creencia imposibilita la del otro se hace impensable una forma en la que todos los miembros de esa sociedad puedan convivir.

En el pluralismo, sin embargo, necesitamos valores comunes que den base a lo que pudiésemos constituir como democracia, en donde cada uno de los miembros de la sociedad se sienta cobijado y respetado en su libertad individual (Román, 2000). El pluralismo permite una convivencia real de los miembros sin dejar de lado que esto implica un desafío (Vargas, 2013). Esto se debe principalmente a que en el pluralismo se deben considerar la diversidad y, en algunos casos, divergencias respecto de lo que se considera "vida buena» (Cortina, 2010; Vargas, 2013; Vásquez, 2011). Sin embargo, los mínimos morales de los que se hablaba anteriormente nos sirven como marco de convivencia para todos los miembros de las sociedades pluralistas actuales. Es decir, se establece un marco referencial que evita la anarquía valórica en la que cada uno de los sujetos establece sus propios parámetros. En el caso del pluralismo, al generar valores de respeto al otro se limita el surgimiento de valores que impidan la convivencia de los miembros de la sociedad. Las libertades individuales siguen manteniéndose, pero con una actitud de tolerancia, entendiéndose a la tolerancia como la aceptación del otro en su forma de concebir el mundo y su visión respecto de éste (Cortina, 2000).

Por otro lado, existen riesgos en las sociedades plurales democráticas de Occidente que surgen a la par con el desarrollo de la ciencia y la técnica. La ética tradicional occidental responde a este desarrollo del hombre y sus sociedades en función de la técnica, así como también supone marcos conceptuales opresivos 
(Moreno, 2016). Se le considera una era antropocéntrica y "próxima", es decir, dedicada a regular las relaciones entre los hombres ya fuese en sí mismos o con los más cercanos (Cortina, 2000). En este sentido, la apuesta ética en la sociedad de la información y la comunicación es el desarrolllo de una moral plural que sostenga el devenir de una sociedad intercultural, diversa e inclusiva (Cortina, 2003; Vásquez, 2011; Cerdán, 2013).

\section{El DiÁlOGO COMO FUNDAMENTO DEMOCRÁTICO EN LA ESCUELA}

Teniendo en mente lo comentado anteriormente, el diálogo se convierte en la base del desarrollo democrático en la escuela, pues en ella se encuentran los distintos puntos de vista respecto de la política, la religión, el género, las culturas y las subculturas (Cerdán, 2013). En este sentido, considerando la escuela como una microsociedad, surge la necesidad de comprender la convivencia escolar desde la noción de vivir juntos más que desde la coexistencia, puesto que la escuela implica una cultura pública en donde convive una diversidad de valores (Duhamel y Estivalezes, 2013). Duhamel (2010) señala que el concepto de vivir juntos es polisémico, sin embargo, se caracteriza por la creación de un espacio común armónico, en donde dicha armonía se produce a través del diálogo. Esta conceptualización del diálogo debe ser entendida como herramienta intercultural que permite la generación de espacios comunes, cuyos efectos se extienden más allá de los centros escolares, por lo que la discusión respecto del diálogo en la escuela conlleva tanto un debate ético como político (Duhamel y Estivalezes, 2013). En este sentido, el reconocimiento del multiculturalismo al interior de la escuela y del diálogo como mediador intercultural implica aceptar el pluralismo de las sociedades occidentales actuales (Coca y López de la Fuente, 2016).

A pesar de la magnitud del debate, es posible observar que el diálogo no ocupa gran parte del quehacer escolar. De hecho, se evita, generando resistencia y tensión en la relación profesor-alumno, o alumno-escuela (Llaña y Escudero, 2001). Por ejemplo, durante los años 2006 y 2011, se generaron movimientos estudiantiles potentes y respaldados por la ciudadanía chilena, en los que los alumnos y alumnas manifestaron su desacuerdo respecto del proyecto educativo de país, que es inexistente a la fecha, entre otros temas como la gratuidad de la educación (Fleet, 2011; Fernández, 2013; Valdebenito, 2013; Valderrama, 2013). Uno de los puntos discutidos residía en la forma en la que las decisiones sobre las políticas públicas de educación eran tomadas por las autoridades, así como la operacionalización de las mismas por las instituciones escolares y de Educación Superior. Además de demostrar el quiebre evidente del sistema y sus actores, esta queja pública a través de tomas de centros educativos y universidades demostró la ineficiencia del sistema para hacerse cargo de la discusión de las ideas y de la capacidad de generar consensos a través del diálogo.

Por otro lado, y prestando atención a la cotidianidad del mundo escolar, es posible observar acciones violentas al interior de los centros escolares, de las cuales 
somos testigos silenciosos detrás de una pantalla de televisión. Estas acciones violentas ocurren constantemente entre los mismos estudiantes (Small et al., 2013; Méndez y Cerezo, 2015); en contra de los profesores (Terry, 1998; Espelage et al., 2013; McMahon, 2014), o desde los mismos profesores hacia sus estudiantes, lo que incluye los medios digitales (Kowalsky et al., 2012; Kyriacou y Zuin, 2016). Sin duda, este fenómeno deja de manifiesto un síntoma de una relación constreñida por el escaso diálogo. Consecuentemente, es posible asegurar que los espacios comunes de respeto por el otro y su diferencia, así como la poca generación de transacciones de significados, se han descuidado. Entonces, es crucial establecer instancias de diálogo capaces de acoger lo verbal y no verbal en donde primen la escucha y el encuentro, estableciendo relaciones veraces de confianza y de interpelación (Mínguez, Romero y Pedreño, 2016).

Otro ejemplo más del escaso diálogo, pero esta vez a nivel curricular, en Chile es la pérdida del Consejo de Curso como espacio de discusión. Esta actividad curricular comenzó en liceos experimentales del país a mediados del siglo xx y se consolidó en los sesenta (Nervi y Nervi, 2007; Núñez, 2002). En dicha asignatura, los alumnos organizaban sus propios contenidos y regulación respecto de sus intereses, eligiendo democráticamente directivos de curso, estableciendo metas y tópicos a tratar. Sin embargo, hoy en día ese espacio mantiene sólo su nombre, debido a que se utiliza como comodín para las actividades que la escuela determine necesarias o para realizar actividades extraprogramáticas. Por lo tanto, cuando la escuela no determina qué hacer, los alumnos deben hacerse cargo de aquel que era su espacio, en el que, lamentablemente, la gran mayoría de ellos no sabe qué hacer, y prefieren utilizar el tiempo destinado al Consejo para realizar tareas atrasadas o preparar presentaciones para "asignaturas de mayor peso" (Nervi, 1999).

A pesar de ello, aquellas conductas también tienen un carácter dialógico, en el sentido de que expresan, explícitamente o no, el descontento de los alumnos. Para Llaña y Escudero (2001), estas conductas son una forma de comunicación pues la resistencia es también un mensaje claro hacia el otro. En otras palabras, la resistencia comunica y urge a la respuesta de parte de un interlocutor. Por lo tanto, la escuela y los profesores deben ser capaces de escuchar en cuanto actores implicados en el diálogo.

Claramente, la escuela no está acostumbrada a dialogar, sobre todo en la Educación Media. Como se lee al comienzo, la escuela intenta persuadir y, en algunos casos, dominar con las «verdades» que considera cruciales para el desarrollo de los estudiantes, sin ningún atisbo de relación a su cultura e identidad juvenil (Posada, 2014). Por lo tanto, el diálogo se ve aún más dañado por la poca disposición a generar espacios dialógicos, considerando las diferencias generacionales y culturales. Por otro lado, no se ha de olvidar que las sociedades se han acostumbrado a convivir evitando poner atención a la subyugación y la opresión (Schöem y Hurtado, 2001; Moreno, 2016), y, por tanto, es posible asegurar que se han olvidado además de dialogar con su propia historia. En este sentido, nos encontramos con un desafío pues la sociedad ha dialogado desde la homogeneidad, no desde 
la heterogeneidad, lo que ha perpetuado el sistema de valores imperante en las sociedades occidentales (Schöem y Hurtado, 2001) y permeado la institución escolar. En este sentido, se mantienen a través de los esterotipos culturales de la escolarización (Martin y Nakayama, 2010; Schöem y Hurtado, 2001) y se presentan también como una resistencia. Sin embargo, la escuela debería ser la primera en dar el paso, especialmente cuando los efectos de este escaso diálogo se manifiestan en violencia concreta al interior de los centros escolares, sin incluir los efectos de la llamada violencia simbólica (Bourdiue, 1987). En este sentido, el rol del docente es fundamental como agente de intervención y prevención de aquellas conductas violentas (Demaray et al., 2013; Veenstra et al., 2014; Bradshaw et al., 2013; Guimond et al., 2015).

Pero cómo generar espacios de diálogo en un contexto de resistencia. La respuesta parece radicar en la comprensión de la resistencia como mensaje y parte del diálogo entre los actores, por lo que las acciones educativas deberían ser orientadas hacia el diálogo. En este sentido, una educación en valores pluralistas, basada en el diálogo, permitiría crear instancias de escucha entre los miembros de una comunidad educativa, validando a sus miembros como interlocutores o, como dirían Mínguez, Romero y Pedreño (2016), permitiendo que el educador y el educando se vinculen vitalmente. Sin embargo, esto no deja de ser un reto, porque, así como los mismos alumnos manifiestan resistencias, los profesores y directivos de un centro también lo hacen. Es, entonces, necesario un cambio profundo en la forma de concebir los proyectos educativos y sus planes de acción. Implica que los actores se involucren de lleno en la planeación y en la aplicación de sus metas. Esas instancias permiten el diálogo desde el comienzo, en otras palabras, desde los principios que fundamentan una escuela.

\section{LA CUESTIÓN CURRICULAR}

En nuestro país, la Reforma Curricular de los 90,y sus posteriores ajustes del año 2009 y 2015, consideró distintos objetivos y contenidos mínimos para las escuelas públicas. Dentro del currículo de los 90 se encuentran los denominados Objetivos Fundamentales Transversales (OFT), que guardan relación con el desarrollo de actitudes y valores en los alumnos, los que deberían afrontarse en cada contenido curricular disciplinar (García-Huidobro, 1998). Nervi (1999) los categoriza como «una explicitación que no tiene precedentes en Chile, de las orientaciones valóricas comunes que deben permear la experiencia escolar, y, por tanto, una respuesta a los requerimientos de mayores capacidades creadoras de sentido y de socialización que la sociedad de fines de siglo le plantea a la institución escolar» (p. 50). En sus supuestos, la educación en valores cobra gran importancia, y en ello, la capacidad de dialogar entre diversas culturas y credos. En este sentido, los OFT se transforman en una directriz para la orientación de una escuela pluralista y lo que ésta debería procurar, en donde dichos objetivos juegan un papel central tanto en la definición curricular en los distintos niveles del sistema escolar como en el rol de la escuela. 
Sin embargo, se puede apreciar a simple vista que los conflictos estudiantiles de la útima década nos dan cuenta de un fenómeno que nada tiene que ver con la creación de sentidos y, aun así, este sinsentido expresado o no por alumnos y profesores nos da cuenta de un diálogo truncado, que sin embargo comunica. De hecho, la casi nula valoración del Consejo de Curso, lugar de diálogo entre las diferencias por naturaleza, parece estar obsoleto. Es quizás que las expectativas de democracia desde la verticalidad de los aparatos administrativos y de la política pública no se condicen con las de los estudiantes, lo que indudablemente refleja el poco contacto e intercambio de ideas entre los actores. Es, entonces, urgente una verdadera articulación de los OFT en las aulas chilenas bajo la creación de instancias de diálogo entre los sujetos.

Para esta difícil tarea es importante la vinculación. Al principio de este trabajo se comentaba la riqueza interpretativa del término y, en ello, se comentaba la especial semanticidad vinculante del diálogo, en el que somos capaces de relacionarnos y establecer afectos entre los seres humanos. La escuela no reconoce necesariamente la importancia de los afectos. Muchas veces prevalece únicamente la transmisión disciplinar en el aula, la normativización de las conductas en los recreos, la estandarización del alumnado sin considerar su expresión. El escuchar y el establecer relaciones afectuosas permiten un mejor diálogo, pero para ello se debe permitir también la expresión del otro sin prejuicios y en donde los actores dialogantes puedan expresarse desde su otredad (Águila, 2004; González Jiménez et al., 2015; Kaplan, 2015). En este sentido, la escuela, si no es capaz de escuchar esta diferencia entre sus sujetos, difícilmente se podrá vincular con sus sujetos. Es por ello que a los estudiantes les parece que la escuela es una instancia detenida en el tiempo, incapaz de adaptarse a los requerimientos y experiencias de sus alumnos (Llaña y Escudero, 2001). Es decir, la escuela no se vincula ni vincula a los miembros de su comunidad, porque no dialoga. Es aquí donde debemos comenzar a romper ciertos consensos del discurso pedagógico. Para que la escuela se transforme en una instancia dialogante, democrática y pluralista, debe romper con el mecanicismo y el academicismo. Debe ser capaz de interpretar las necesidades de sus miembros y saber dar respuesta a las suyas propias a través de la negociación de significados que supone el diálogo.

Dentro de los OFT existe la posibilidad curricular de hacerlo. Es sabido que la praxis no se condice necesariamente con las directrices administrativas ministeriales (Hargreaves, 2003), sin embargo, al menos parece existir preocupación al respecto. La utilización de los OFT en una adecuada educación en valores basada en el diálogo podría fomentar aún más el diálogo. A través del diálogo se podrían crear los ambientes y experiencias necesarios para una mayor vinculación con el proyecto educativo y la misma formación de los alumnos. Una de ellas puede ser el mismo análisis de los conflictos que un determinado curso pueda tener. Pérez Pérez (1999) asegura que las situaciones conflictivas que van surgiendo en el aula como fruto de la convivencia diaria, si se aprovechan adecuadamente, constituyen un elemento pedagógico muy valioso para generar cambios positivos en las 
convicciones morales de los alumnos. El reconocer experiencias vividas por los mismos alumnos toca la propia emocionalidad, lo que transciende en una suerte de metodología que involucra tanto lo cognitivo como lo afectivo, entrelazando estos aspectos, haciendo que la experiencia de diálogo sea más significativa, porque "cuando lo cognitivo y lo afectivo quedan vinculados por la actividad del sujeto se dobla su eficacia y persisten en la memoria sus logros» (Águila, 2004, 32). Es decir, por una parte, se contribuye a la educación de las emociones y, por otra, permite que el mismo análisis respecto del diálogo perdure en el tiempo y se convierta en aprendizaje. La escuela en ocasiones olvida educar las emociones de sus alumnos en donde los currículos siguen atendiendo a disciplinas del saber que poco y nada se conectan con las vivencias de sus alumnos, por lo tanto, cualquier esfuerzo de diálogo con las disciplinas no involucra a los sujetos. Como se decía anteriormente, nos hemos quedado en una mera transmisión de cultura sin posibilitar las capacidades creativas de los sujetos para transformarla.

\section{RECONSTRUYENDO EL DiÁLOGO}

Es vital, entonces, a modo de síntesis, una exploración de la cultura juvenil, "escuchando" a los alumnos, palpando sus experiencias y permitiéndoles expresarse, educando asimismo la emocionalidad de los estudiantes y generando capacidad de escucha.

Sin embargo, primeramente, debemos considerar ciertos aspectos fundamentales para establecer el diálogo. Uno de ellos es «mostrar un deseo genuino por participar, sin importar si habrá algo productivo después de ello» (Nava, 1999, 3). Aquí nos encontramos con dificultades o resistencias de parte de los miembros de un centro educativo. Por ejemplo, los profesores no siempre están dispuestos a escuchar a sus alumnos y, menos aún, a dialogar con ellos. Los mismos alumnos, por otra parte, tampoco quieren hacerlo porque sienten que no se les va a escuchar (Llaña y Escudero, 2001). En consecuencia, la escuela y los profesores deben dar el primer paso, creer en la capacidad dialogante de los estudiantes, pues son estos actores quienes intencionan el acto educativo y, por tanto, el diálogo entre los actores.

Por otro lado, esa misma necesidad de escucha que los estudiantes expresan en sus resistencias no significa que los alumnos pretenden conseguir un resultado que los beneficie exclusivamente a ellos. Pensar que el diálogo está teleológicamente dirigido hacia producir alguna respuesta a favor de alguna de las partes sería erróneo. Si así fuese, estaríamos atribuyéndole una característica de mecanismo para conseguir lo que se quiere. El diálogo no tiene otro fin que el comunicarnos, expresarnos, compartir las experiencias vitales propias con las del otro, respetando la diferencia, creando espacios comunes de convivencia (Nava, 1999). Un diálogo unívoco, en donde no se intercambien significados, y que asimismo se espere prevalecer sobre el otro, no constituye un diálogo real. La necesidad de un consenso es lo que diferencia al diálogo del debate. En el debate siempre se busca 
un ganador, independientemente de si se trata de la capacidad argumentativa del orador o el impacto en el otro, su fin es conseguir derrotar al otro a través de la palabra. No negocia sus significados. Por otro lado, el diálogo implica la interacción cara a cara en donde se requiere la capacidad de expresarnos directamente sobre aquello en que nos vemos afectados. Indudablemente, este requisito para el diálogo sugiere que la misma escuela enuncie los discursos respecto de sus estudiantes como elementos constituyentes de una relación dialógica frontal, en otras palabras, debería partir por encarar sus demandas en abierta posición de escucha. Eso implica que los profesores y directivos deben «estar a la escucha y plenamente en atención del significado de las palabras» (Nava, 1999, 3), es decir, enfrentados honestamente a la expresión de conceptos, visiones de mundo, creencias e ideologías de los alumnos y alumnas de manera tal de evitar un diálogo falso desde la administración (Guo-min, 2013). Sin embargo, la iniciativa intencionada desde la escuela no libera a los estudiantes, sino que, lo contrario, les exige un compromiso en tanto actores y parte del diálogo, pues la negociación de nuestros propios pensamientos nos involucra, nos permea y convierte la relación dialogante en un sitio común para la expresión, la comunicación y la transacción de otros puntos de vista. En definitiva, el diálogo "contribuye de una manera decisiva en la formación de personas tolerantes y facilita la convivencia escolar» (Pérez Pérez, 1999, 119). Consecuentemente, no sólo permite la creación de ambientes saludables en la escuela, sino que los prepara para ejercer futuras relaciones de convivencia sanas en la sociedad.

El fomento del diálogo como estrategia de convivencia permite que los estudiantes aprendan a escuchar de una manera activa a los demás y reelaborar su propio pensamiento y valoraciones, que a partir de ese momento estarán influidas por la información recibida de los otros (Pérez Pérez, 1999). Es decir, esta escucha en el diálogo permite generar nuevo pensamiento, nuevas estructuras cognitivas modificables de acuerdo a la relación que el sujeto ejerce con el mundo y los otros (Skidmore y Murakami, 2016). Permite que el sujeto pueda establecer criterios o juicios de valor en relación a la convivencia sana en la diferencia, aprendiendo de esa relación dialógica.

Al parecer, los beneficios del diálogo no sólo se manifiestan en el desarrollo de habilidades de escucha y de tolerancia, sino que, además, permite el aprendizaje de otras culturas, visiones de mundo, para enriquecer el propio mundo interior, ya sea afectivo o cognitivo. El valor, entonces, del diálogo es cuantioso debido a que las salas de clases hoy en día son variopintas, entretejiéndose una multiplicidad de culturas y subculturas producto de las migraciones y de la convivencia con tribus urbanas juveniles (Dervin, 2015; Fuentes, 2016).

Finalmente, reconstruir el diálogo suscita una gran incertidumbre en una sociedad como la chilena, la que lleva consigo los resultados de su historia, de la oposición al diálogo, de la no escucha, de la muerte por la libertad de expresión, y es la de no estar seguros de si estamos preparados para dialogar. Es decir, nuestro país no se ha dispuesto a dialogar con su propia historia. Por tanto, nuestras 
escuelas deberían fomentar el diálogo en todos sus sentidos, de manera tal que las nuevas generaciones sean capaces de escucharse, de vincularse y de creer en el otro.

\section{CONSIDERACIONES FINALES}

Como se aprecia en los apartados anteriores, el diálogo se construye a través de la capacidad de escucha, de la aceptación del otro como un interloctor válido y comprendiendo las resistencias como un acto comunicativo. Por tanto, es necesario que la escuela preste atención a estos fenómenos y sea capaz de interpretarlos como significados, lo que requiere de la validación de las resistencias como mensajes válidos. En este sentido, a través de la intencionalidad de la enseñanza, la escuela debe hacerse cargo de generar espacios de diálogo al interior de la escuela no sólo por el hecho de permitir una mejor convivencia o clima escolar, sino también por la trascendencia del aprendizaje del diálogo en un ambiente resguardado y que afronta a pequeña escala las interacciones sociales.

Las necesidades de la sociedad en nuestros días requieren una capacidad de escucha a las distintas culturas que en ella conviven, y la escuela presenta una posibilidad para la construcción del diálogo intercultural en contextos de infancia y juventud. Estos espacios dialógicos construyen un lugar de encuentro intercultural, enriquecen la comprensión de lo intercultural y fomentan el acercamiento al otro a través de la disminución de la desigualdad de condiciones culturales (Vila, 2014). En consecuencia, la creación de instancias de diálogo contribuye a la vinculación con el otro y disminuye los desencuentros interculturales.

Según Plante (2013), la comprensión del otro y su subjetividad requiere del diálogo y el acuerdo intersubjetivo. Es por ello que la utilización del diálogo como una estrategia enseñable en los contextos escolares permite una mejor convivencia para la diversidad y de respeto a las diferencias individuales en un contexto saludable y cimenta las futuras maneras de enfrentarse a situaciones de conflicto que tensionan la comunicación y la solución de problemas. Por otro lado, permite valorar al otro en su otredad al aceptarlo como interlocutor, lo que trasciende en una comunicación más simétrica entre los actores, para así comprender el valor de la comunicación como un proceso abierto y de construcción colectiva. Además, como afirman Mínguez, Romero y Pedreño (2016), supone salir de uno mismo para vincularse al otro, para estar juntos, lo que repercute en la relación entre el educador y el educando y se transforma en, según denominan los autores, una pedagogía del otro. En suma, la escuela debe propender a la escucha e interpretar las tensiones de la cultura escolar que le son propias y cotidianas para aprender a vivir juntos.

Indudablemente, los centros escolares han de comprenderse a sí mismos como sistemas complejos y heterógeneos, en donde tanto la administración como los profesores, estudiantes y otros miembros de la escuela se encuentran y validan mutuamente como interlocutores y mantienen una relación dialógica. A pesar de que las responsabilidades sobre el diálogo como estrategia de convivencia y 
de interacción cotidiana recaen en todos aquellos que participan de la comunidad escolar, es la escuela -su administración y cuerpo docente- quien debe dar el primer paso para acercar posiciones. Es decir, las instituciones escolares han de hacerse cargo del carácter intencional de la acción educativa. En consecuencia, la escuela debe dar pie a la enseñanza de valores democráticos como la diversidad, la tolerancia y la pluralidad (Coca y López de la Fuente, 2016). Evidentemente, esto supone un camino difícil y de transacciones constantes de la autoridad y del rol controlador que generalmente ejerce la escuela, por lo que es necesario desvelar el estado en el que se encuentran las posiciones para, finalmente, poder llevar a cabo estrategias concretas que permitan establecer la comunicación y el diálogo al interior de los centros escolares.

\section{REFERENCIAS BIBLIOGRAFICAS}

Águila, E. (2004) Educar en valores en sociedades y escuelas pluralistas. Perspectivas Éticas, 8, Centro de Estudios de Ética Aplicada, Facultad de Filosofía y Humanidades, Universidad de Chile.

Bohm, D. (1997) Sobre el diálogo. Barcelona, Kairós.

Bourdieu, P. (1987) Distinction: A social critique of the judgment of taste (R. Nice, Trans.). Cambridge, MA, Harvard University Press.

Bourdieu, P. (2011) The forms of capital (1986). En Szeman, I. y Kaposy, T. Cultural theory: An anthology, 81-93.

Bradshaw, C. P.; Waasdorp, T. E.; O'Brennan, L. M. y Gulemetova, M. (2013) Teachers' and education support professionals' perspectives on bullying and prevention: Findings from a national education association study. School psychology review, 42 (3), 280.

CAREY, J. (1989) Communication as culture: Essays on media and society. Boston, Unwin Hyman.

CERDÁn, L. L. (2013) La integración escolar. Revista de Claseshistoria, (6), 6.

Coca, J. R. y López de la Fuente, G. (2016) Reflexiones sobre pluralismo político y educación intercultural en las sociedades democráticas contemporáneas. Aposta. Revista de Ciencias Sociales, 70, 132-154.

Cortina, A. (2000) Ética mínima. Introducción a la Filosofía Práctica. Madrid, Tecnos.

Cortina, A. (2003) Pluralismo moral. Ética de mínimos y ética de máximos. Perspectivas Éticas, 7, Centro de Estudios de Ética Aplicada, Facultad de Filosofía y Humanidades, Universidad de Chile.

Cortina, A. (2010) Problemas éticos actuales. Revista Taula. Quaderns de pensament, (42), $173-185$.

Demaray, M. K.; Malecki, C. K.; Secord, S. M. y Lyell, K. M. (2013) Agreement among students', teachers', and parents' perceptions of victimization by bullying. Children and Youth Services Review, 35 (12), 2091-2100.

Dervin, F. (2015) Towards post-intercultural teacher education: analysing «extreme» intercultural dialogue to reconstruct interculturality. European Journal of Teacher Education, 38 (1), 71-86.

Duhamel, A. (2010) Le «vivre-ensemble»: la citoyenneté et le politique entre conflit et confiance, en JuTRAs, F. (ed.) L'éducation à la citoyenneté: enjeux socioéducatifs et pédagogiques. Québec, Qc, Presses de l'Université du Québec, 111-130. 
Duhamel, A. y Estivalezes, M. (2013) Vivre ensemble et dialogue: du programme québécois d'éthique et culture religieuse à la délibération démocratique. McGill Journal of Education / Revue des sciences de l'éducation de McGill, [S. 1.], 48 (1), 79-98.

espelage, D.; Anderman, E. M.; Brown, V. E.; Jones, A.; Lane, K. L.; McMahon, S. D. y ReyNOLDS, C. R. (2013) Understanding and preventing violence directed against teachers: Recommendations for a national research, practice, and policy agenda. American Psychologist, 68 (2), 75.

FERnánDEZ, J. (2013) La protesta social en Chile (2006-2011): Conflicto social y repertorios de acción en torno a los movimientos estudiantil, mapuche y ambiental. Documentos promovido por el Grupo de Investigación en Gobierno, Administración y Políticas Públicas. Programa de Doctorado en Gobierno y Administración Pública. Instituto Universitario de Investigación Ortega y Gasset, Madrid.

FleEt, N. (2011) Movimiento estudiantil y transformaciones sociales en Chile: una perspectiva sociológica. Polis, 10 (30), 1-13. doi:10.4000/polis.2152.

Fuentes, J. L. (2016) Cultural diversity on the Council of Europe documents: The role of education and the intercultural dialogue. Policy Futures in Education, 1478210316630467.

García-Huidobro, J. E. (ed.) (1998) La reforma educacional chilena. Santiago, Ediciones Popular.

Guimond, F. A.; Brendgen, M.; Vitaro, F.; Dionne, G. y Boivin, M. (2015) Peer victimization and anxiety in genetically vulnerable youth: The protective roles of teachers' selfefficacy and anti-bullying classroom rules. Journal of abnormal child psychology, 43 (6), 1095-1106.

Guichard, J. (2013) Comment aider les jeunes formés dans une école malade de l'orientation à s'orienter dans la vie? L'orientation scolaire et professionnelle, 42 (2), 181-198.

González Jiménez, J.; Gutiérrez, A. V.; Moreno, D. P.; Sánchez, L. N.; Sierra, J. A. y Zapata, J. G. (2015) Huellas discursivas de otredad en ámbitos de violencia escolar. PAPELES, 6 (12-13), 33-46.

Guo-Min, C. H. E. N. (2012) Exploration of Fake Dialogue Phenomenon in School Management. Jiangsu Education Research, 10, 005.

Hargreaves, A. (2003) Teaching in the Knowledge Society: Education in the Age of Insecurity. New York, NY, Teachers College Press.

Kaplan, C. V. (2015) Cuidado y otredad en la convivencia escolar: una alternativa a la ley del talión. Pensamiento Psicológico, 14 (1).

Kowalski, R. M.; Limber, S. P.; Limber, S. y Agatston, P. W. (2012) Cyberbullying: Bullying in the digital age. John Wiley y Sons.

Kyriacou, C. y Zuin, A. (2016) Cyberbullying of teachers by students on YouTube: challenging the image of teacher authority in the digital age. Research Papers in Education, 31 (3), 255-273.

Llaña, M. y Escudero, E. (2001) Alumnos y profesores: radiografía de un desencuentro. Santiago, Departamento de Educación, Universidad de Chile.

Mcmahon, S. D.; Martínez, A.; Espelage, D.; Rose, C.; Reddy, L. A.; Lane, K. ... y Brown, V. (2014) Violence directed against teachers: Results from a national survey. Psychology in the Schools, 51 (7), 753-766.

Martin, J. y Nakayama, T. (2010) Intercultural communication in context. New York, McGraw Hill.

MÉndez, I. y Cerezo, F. (2015) Bullying y factores de riesgo para la salud en estudiantes de secundaria. European Journal of Education and Psychology, 3 (2). 
Mínguez, R.; Romero, E. y Pedreño, M. (2016) La pedagogía del otro: bases antropológicas e implicaciones educativas. Teoría de la Educación. Revista Interuniversitaria, 28 (2), 163-183.

Moreno, D. P. T. (2016) Éticas ecofeministas: la comunidad de la vida. Cuadernos de Filosofía Latinoamericana, 37 (114), 15.

NAva MelÉndez, W. (1999) El diálogo en la educación holista: breves reflexiones sobre su aplicación en el aula. Revista de Educación/Nueva Época, (11).

Nervi, M. L. (1999) La Reforma Curricular Chilena. Santiago, LOM.

Nervi, M. L. y Nervi, H. (2007) ¿Existe la pedagogía? Hacia la construcción del saber pedagógico. Santiago, Editorial Universitaria.

Núñez, I. (2002) La formación de docentes. Notas históricas, en Avalos, B. Profesores para Chile: Historia de un Proyecto. Santiago, Ministerio de Educación.

Plante, M. (2013) Peut-on enseigner le vivre-ensemble? Mc Gill Journal of Education / Revue des sciences de l'éducation de McGill, [S. 1.], 48 (1), 155-164.

PÉrez Pérez, C. (1999) Educación para la convivencia como contenido curricular: propuestas de intervención en el aula. Estudios Pedagógicos, 25, 113-130.

Posada, J. G. (2014) Mínimos valorales para la convivencia de los jóvenes (Tesis de Máster). Universidad de Manizales, Colombia.

Rodríguez, J. J. M. y Peralta, M. I. R. (2013) Ética y escuela. Fundación Europea para el Estudio y Reflexión Ética.

Román, B. (2000) Los retos de la ética en el nuevo milenio. Ars Brevis, (6), 311-336.

Schoem, D. y Hurtado, S. (2001) Intergroup dialogue: deliberative democracy in school, college, community, community and workplace. Michigan, Ann Arbor The University of Michigan Press.

Skidmore, D. y Murakami, K. (eds.) (2016) Dialogic Pedagogy: The Importance of Dialogue in Teaching and Learning (vol. 51). Multilingual Matters.

Small, P.; Neilsen-Hewett, C. y Sweller, N. (2013) Individual and Contextual Factors Shaping Teachers' Attitudes and Responses to Bullying among Young Children: Is Education Important? Asia-Pacific Journal of Research in Early Childhood Education, 7 (3).

TERrY, A. A. (1998) Teachers as targets of bullying by their pupils: A study to investigate incidence. British Journal of Educational Psychology, 68 (2), 255-268.

VAldebenito, M. J. (2013) ix encuesta Cide: El diagnóstico a la justicia y equidad en la educación chilena. Mensaje, 62 (618), 48-49.

VAlderrama, L. B. (2013) Jóvenes, Ciudadanía y Tecnologías de Información y Comunicación. El movimiento estudiantil chileno. Revista Latinoamericana de Ciencias Sociales, Niñez y Juventud, 11 (1), 123-135. doi:10.11600/1692715x.1117010812.

Vargas, J. O. (2013) Pedagogía y ética en la construcción de ciudadanía: la formación en valores en la educación comunitaria. Revista Contexto \& Educação, 18 (70), 47-62.

VÁsquez, G. H. (2011) Educación y ética para una ciudadanía cosmopolita. Revista Iberoamericana de Educación, (55), 191-203.

Veenstra, R.; Lindenberg, S.; Huitsing, G.; Sainio, M. y Salmivalli, C. (2014) The role of teachers in bullying: The relation between antibullying attitudes, efficacy, and efforts to reduce bullying. Journal of Educational Psychology, 106 (4), 1135.

Vila Merino, E. (2014) Cartografías interculturales: procesos educativos y traducción entre culturas. Teoría de la Educación. Revista Interuniversitaria, 25 (2), 69-87. 
